\title{
Resin Coating Technique for Protection of Pulp and Increasing Bonding in Indirect Restoration
}

\author{
Toru Nikaido $^{1}$ - Go Inoue ${ }^{1}$ Tomohiro Takagaki ${ }^{1}$ - Rena Takahashi ${ }^{1}$ - Alireza Sadr $^{2,3}$. \\ Junji Tagami ${ }^{1}$
}

Published online: 9 April 2015

(C) Springer International Publishing AG 2015

\begin{abstract}
A resin coating technique has been widely advocated in recent years. A combination of a dentin bonding system and a flowable composite is applied on the exposed dentin surfaces immediately after tooth preparation and before taking the final impression. The resin coating is beneficial to both minimize pulpal irritation and enhance the bond strength of a resin cement to dentin. Recently introduced thin-film coating materials based on all-in-one adhesive technology are used for resin coating of crown restorations. Thin-film coating materials can be applied in a single clinical procedure and followed by created a barrier-like film layer on the prepared dentin. Therefore, the resin coating
\end{abstract}

This article is part of the Topical Collection on Cariology

Toru Nikaido

nikaido.ope@tmd.ac.jp

Go Inoue

inoue.ope@tmd.ac.jp

Tomohiro Takagaki

takagaki.ope@tmd.ac.jp

Rena Takahashi

renatakahashi@hotmail.com

Alireza Sadr

arsadr@uw.edu

Junji Tagami

tagami.ope@tmd.ac.jp

1 Department of Cariology and Operative Dentistry, Division of Oral Health Sciences, Graduate School of Medical and Dental Sciences, Tokyo Medical and Dental University (TMDU), 1-5-45, Yushima, Bukyo-ku, Tokyo 113-8549, Japan

2 International Exchange Center, Tokyo Medical and Dental University (TMDU), 1-5-45, Yushima, Bunkyo-ku, Tokyo 113-8549, Japan

3 Department of Restorative Dentistry, University of Washington School of Dentistry, 1959 NE Pacific St, Box 357456, Seattle, WA 98195-7456, USA technique may play an important role to protect the dentin physically, chemically, and biologically. The coating materials also have the potential to cover exposed sound enamel and dentin, leading to maximum tooth structure preservation.

Keywords Resin coating technique $\cdot$ Adhesion $\cdot$ Pulp protection $\cdot$ Dentin bonding agent $\cdot$ Indirect restoration . Super dentin

\section{Introduction}

The concept of minimal cavity preparation has become widely accepted for the placement of direct composite restorations with the development of improved materials and adhesive techniques [1]. Although direct composite restorations are the preferred treatment, indirect restorations have advantages over direct techniques for large cavities such as improvements in anatomic form, contour, fracture resistance, and wear resistance [2].

Indeed, cavity preparation for an indirect inlay/onlay restoration is much more aggressive than that of direct composite restoration. For a conventional indirect restoration, intact tooth structure has to be sacrificed to obtain the retention and resistance forms, especially because the conventional luting cements have a poor capability of bonding to tooth structure [3]. Dentin exposed after cavity preparation should be considered as an indirect pulpal exposure since dentin is connected to pulp tissue through dentinal tubules. In indirect restorations, temporary sealing of the prepared cavities has routinely been performed. However, the poor sealing with such a temporary material may easily fail, resulting in accidental exposure of the prepared dentin in the oral cavity, causing irrigation from physical, chemical, and/or bacterial stimuli $[4,5]$. Therefore, the exposed dentin should be protected immediately after the preparation. 
The sealing property of modern dentin adhesive systems is superior to those of the conventional temporary sealing materials [6]. A recent dentin bonding system has good biocompatibility to pulp tissue [7].

\section{Resin Coating Technique}

A resin coating technique was proposed in the early 1990s in order to protect the exposed dentin surface after cavity preparation for indirect restorations [8, 9]. The clinical procedures of the resin coating technique are illustrated in Fig. 1a. For the resin coating technique, a combination of a dentin bonding system and a flowable resin composite was applied on the preparation just after cavity preparation and before taking the final impression, producing a hybrid layer and tight sealing film on the dentin surface [10]. The resin coating technique allows for protection and coverage of the prepared dentin immediately after cavity preparation reducing postoperative sensitivity [11]. In addition, the resin coating can enhance dentin bonding strength of a resin cement and provide good interfacial adaptation and marginal seal $[12,13]$. Paul et al. and Bertschinger et al. reported "dual application" [14, 15]. Magne et al. proposed "immediate dentin sealing (IDS)" $[16,17]$. These ideas are believed to share a similar concept with the proposed resin coating technique.

\section{Selection of Dentin Bonding System as a Resin Coating Material}

Nowadays, a lot of different types of dentin bonding systems are available in the market. These dentin bonding systems are categorized according to the design and mechanism of the adhesive systems. However, not all these adhesive systems are considered to be suitable for use of the resin coating technique. In general, contemporary dentin bonding systems can be classified into two main categories: self-etching systems and acid etching systems. However, the category of selfetching systems itself consists of two subcategories, namely the two-step self-etch and the one-step self-etch systems. The latter is also called the "all-in-one" adhesive. More, recently, universal adhesives have been introduced to the market, which can be categorized under the all-in-one adhesives in terms of their mechanism. The category of acid-etching or etch-andrinse systems included the conventional phosphoric acid etching in either three-step etching, priming and bonding systems, or two-step etching and priming/bonding systems.

In the case of two-step self-etching systems, there are two components in two separate bottles of the self-etching primer and the bonding agent. One or several acidic monomers are included in the components of the self-etch primer, along with water and solvents. The self-etch agent can perform both etching or conditioning and priming functions simultaneously. On the other hand, in the case of all-in-one systems, the roles of the two agents are combined into a single application step.

The 30-40\% concentration of phosphoric acid in the etchand rinse-systems removes the smear layer from a prepared dentin surface and widens the tubule orifices of the dentin surface, demineralizing dentin and exposing the collagen fibrils over a depth of 3-5 $\mu \mathrm{m}$ [18]. In this regard, phosphoric acid etching results in severe demineralization of dentin surface when compared to the self-etching systems, particularly milder systems that have a $\mathrm{pH}$ of around 2.0 and higher. Moreover, in the selection of the adhesive system as a resin coating material, protection of dentin and pulp tissue is very
Fig. 1 Clinical procedures of the resin coating technique

$\mathbf{a}$

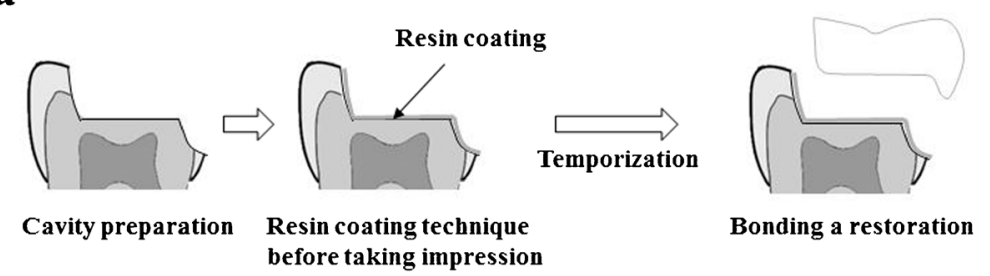

b

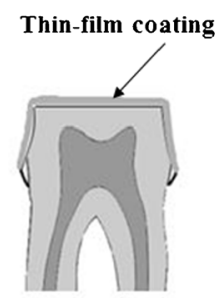

Full crown preparation c

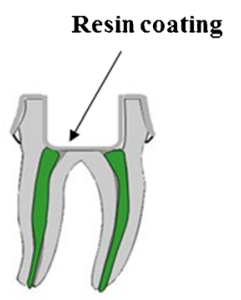

Endodontically treated tooth d

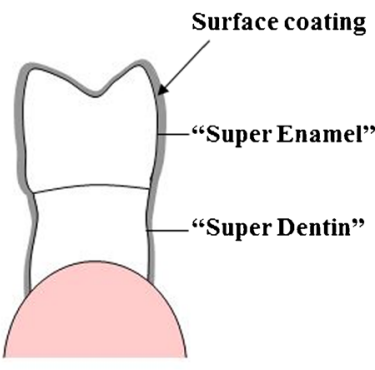

Reinforced tooth, "Super Tooth" 
important. Therefore, self-etching adhesive systems are the preferred materials for the resin coating technique rather than an acid-etching adhesive system.

\section{New Concept of Dentin Bonding Mechanism}

Dentin bonding systems have been simplified and improved dramatically during the past decades. Hybridization concept was proposed as a dentin bonding mechanism by Nakabayashi et al. [19]. In this concept, monomer penetration into dentin and its polymerization in situ creates a hybrid layer, which is believed to be essential for good dentin bonding [19]. However, a new zone, the so-called acid-base resistant zone (ABRZ), was found beneath the hybrid layer [20]. The formation of ABRZ has been confirmed only with a selfetching system, but not with an acid-etching system [20-22].

The self-etch adhesive systems demineralize dentin mildly and partially, leaving hydroxyapatite crystals in the base of the hybrid layer [23•].Such residual apatite crystals may serve as a template for additional chemical reaction with the functional monomer. Among the functional monomers with different chemical formulae developed for the dentin bonding systems, 10-methacryloxydecyldihydrogen phosphate (MDP) is known to have a high chemical bonding potential to hydroxyapatite forming a very stable bond and excellent water resistance confirmed by the low dissolution rate of its calcium salt in water [24-26].

On the other hand, when the dentin surface is aggressively etched with phosphoric acid, the underlying dentin may become completely demineralized so deep that hydroxyapatite in the underlying dentin would disappear and the bottom of the demineralized dentin would be inaccessible to complete impregnation by the resin [27]. In this case, a functional monomer may not have the opportunity to react with hydroxyapatite at the base of the hybrid layer, resulting in lack of the ABRZ formation with an acid-etching system [23•].

If the ABRZ is assumed made of resin-infiltrated dentin, the same chemical reaction of hydroxyapatite and an acidic monomer in the adhesive may take place in this zone, giving rise to the ability to resist against demineralization from an acid attack from the microorganisms in primary and secondary caries. Therefore, the reinforced dentin was proposed to be called as "Super Dentin," which should be superior to the normal dentin mechanically, chemically, and biologically [28].

\section{In Vitro Evidence for the Benefits of the Resin Coating Technique}

Selection of the coating material influences immediate dentin bonding performance [29] and also dentin bonding durability [30, 31]. A dentin bonding system contains several different ingredients for each adhesive system, influencing its dentin bonding performance and durability. Therefore, selection of dentin bonding systems is the first step for success of the resin coating technique. The combination of the two-step self-etch adhesive and a low viscosity-resin could provide the highest bond strength of cement to dentin $[32,33]$. The additional application of a low-viscosity resin plays an important role in the resin coating technique. A flowable resin composite can protect dentin bonding system from tearing during removal of temporary restoration. It also enhances the adhesive polymerization through the diffusion of its free radicals that polymerize uncured resin in the oxygen inhibited layer $[33,34]$. Moreover, the flowable resin composite layer in the coating technique would prevent possibly adverse interactions that have been reported to occur between residual uncured acidic monomers within the self-etch adhesive and the aromatic tertiary amine derived from chemicaland dual-cured resin composites. In addition, the lowviscosity resin with lower filler content combined with a bonding agent with low modulus of elasticity form a stress-breaking resin layer relieving the polymerization stresses of cement and leading to better adaptation of the resin inlays [34]. The association of these factors has contributed to achieve significantly higher adaptation of resin cements to dentin.

In the dentin bond testing of the bonded specimens, examination of the debonded specimens after tensile bond testing is important. In comparison of the resin-coated specimens with the non-coated specimens, the fracture mode in the resin coated specimens shifted adhesive failure to cohesive failure within the cement [34]. This points out the clinical significance of the resin coating on the sealing of dentin, as even if the restoration fractures.

The use of resin coating technique has been demonstrated to improve overall interfacial sealing of the resin cements. Different methods have been conventionally used to evaluate the marginal integrity and sealing of restorations. The common method is detecting dye penetration depth under a stereoscopic microscope and/or confocal laser scanning microscope (CLSM). However, these conventional methods are considered as destructive methods since they require sample sectioning and may be subjective. More recently, threedimensional and in-depth imaging methods have been introduced and utilized for the characterization of restorations [35•, 36-40]. Optical coherence tomography (OCT) is a noninvasive diagnostic imaging technique that can give real-time, high-resolution images using a safe broadband light source. Imaging of indirect bonded restorations by OCT demonstrated that treatment of dentin surface with resin coating improves long-term interfacial sealing of indirect restorations placed with resin cements $[41 \bullet \bullet]$. 


\section{Clinical Factors Influencing Bonding Properties of the Resin Coating Technique}

Dentin bond strength is affected by many clinical factors [ $[42$, 43]. Just after application of the resin coating, the coating layer has a potential to suffer from various types of contaminations during the succeeding clinical procedures. The material selected for impression making $[9,44]$ and the provisional restoration [9] after application of the resin coating play a pivotal role in the success of the final restoration. The incompletely polymerized resin coating can inhibit the polymerization reaction of impression materials, which depends on selection of the materials $[44,45]$. Removal of the oxygen inhibition layer on the cured coating material prevents interaction of the coating layer with the impression material. In the indirect restorations, the preparations have to be temporarily protected for the patient's functional and esthetic needs. The contamination of the resin coating by the temporary filling material also remains an issue, as it may alter the bond between the existing resin coating and the resin cement $[9,29,46]$. A water-setting temporary material is recommended for temporization for the coated surface because this material does not influence the bond strength to resin-coated cavities $[4,9]$. Sealed dentin surfaces have the potential to bond to resin-based provisional materials and cements. As a result, retrieval and removal of provisional restorations can prove extremely difficult. Tooth preparations must be rigorously isolated with a separating medium (such as water-soluble Vaseline) during fabrication of the provisional restoration. Given the potential exposure of the cured adhesive to the oral fluids as well as the water sorption mechanism, it is recommended to keep the provisionalization period as short as possible up to a maximum of 2 weeks [47, 48].

\section{Thin-Film Coating Materials}

The resin coating technique is applicable to both anterior and posterior bonded restorations. However, the combination of a dentin bonding system and a low-viscosity micro-filled resin creates a thick coating layer on the dentin surface, raising concerns about the thickness of the coating [49]. Thickness of the cured dentin bonding agent can vary significantly according to the composition of adhesive system and also surface geometry [7, 48]. A thick coating is not suitable especially for a crown preparation because of the possibility of deformation of the preparation by the resin coating [49].

Recently, a thin-film coating material has been developed by using the all-in-one adhesive technology, which can create a thin coating layer with less than $10 \mu \mathrm{m}$ in thickness on the dentin surface (see Fig. 1b). An all-in-one adhesive with mild acidity has already been marketed for use as a desensitizing agent for the hypersensitive dentin. Such coating materials are clinically accepted for sealing the exposed dentin of the crown preparation. The thin-film coating material is easily removed after curing even in the gingival sulcus in comparison with the cured adhesive resin in other categories of bonding systems. The thin-film coating material demonstrated to improve the dentin bonding performance of resin cement and prevent marginal leakage beneath the restorations $[5,50,51]$. However, the combinational method of applying a dentin bonding system and a flowable resin composite still provides more reliable dentin bonding performance than the thin-film coating alone.

\section{Application of Resin Coating to the Endodontically Treated Teeth}

The resin coating technique can be applied not only for the preparations of vital tooth but also for root canal dentin of the endodontically treated tooth (as shown in Fig. 1c). The goal of endodontic obturation is to provide an effective apical seal to prevent bacterial invasion from the oral cavity through the root canals. However, the apical seal can be adversely affected by coronal leakage once the coronal restoration is lost or becomes defective [52]. Dissolution of the sealer allows access of periapical fluids, proteins, and bacteria into the root canal. Fractured teeth and leaking/lost temporary restorations are often encountered clinically, which can leave the root canal filling open to the oral cavity. Therefore, the quality of the coronal seal is very important for the ultimate success of any root canal obturation. The application of a resin coating to the coronal opening of the endodontically treated tooth could minimize coronal leakage [52]. Perfect sealing was achieved by coating with a combination of a self-etch adhesive and a flowable resin composite [52].

For successful adhesive restorations of non-vital teeth, it is important to obtain good dentin bonding to endodontically treated dentin. Ariyoshi et al. (2008) demonstrated that resin coating significantly enhanced the microtensile bond strengths of indirect composite cores to pulpal floor dentin [34].

\section{Surface Coating for Tooth Structure Preservation}

Sealing of pit and fissure of occlusal enamel with composite resins has been widely accepted to prevent enamel caries in children and adolescents [53]. Nowadays, approaches of this preventive concept are made to expand to smooth enamel surfaces $[54,55]$. In order to protect enamel from dissolution, the superficial penetration and surface coating of the adhesive and/or caries infiltrant is considered as one of the most optimal treatment options [56]. The MDP functional monomer in the two-step self-etch adhesive 
has shown to resist adjacent enamel at the enamel/ adhesive interface against acid attack [57••]. This fact suggests application of the coating materials can reinforce the tooth surface, "Super Tooth", and contribute to tooth structure preservation (see Fig. 1d). A recent OCT study showed that these resin coating materials could sufficiently protect enamel against aggressive acid challenge [58].

Soft tissue recession because of age, inappropriate toothbrush habits, periodontal disease, or surgical periodontal treatment will cause exposure of susceptible root surface and high incidence of root caries and dentin hypersensitivity [59]. Simple single-visit methods to protect the exposed root surfaces from long-term caries attack are advantageous. However, a promising approach to prevent root caries has not become available yet. Root surface coating with the dentin bonding systems is considered to be an effective measure for protection against caries, erosion, and abrasion [60], as it provides a strong physical barrier with the formation of "Super Dentin" [28]. From the clinical stand point, to control the biofilm adherence on the coating material is also important to reduce caries risk in the oral environment [61]. A series of experimental coating materials with self-cleaning surface property has been developed, which demonstrated such surface property had good potential to inhibit biofilm adherence [62••]. If such materials with a surface property could be combined with the current adhesive technology, the surface coating will become a promising therapy in preventive dentistry in the future.

\section{Conclusions}

The resin coating technique was put into practice with the advance of adhesive materials and technology. For indirect restorations, the exposed enamel and dentin should be sealed with the resin coating technique just before taking impression. The resin coating technique can minimize pulp irritation, improve the bond strength between a resin cement and tooth, and enhance interfacial adaptation of the restorations. This rational approach to adhesion has a positive influence on tooth structure preservation. However, selection of the material for impression making and the provisional restoration after application of the resin coating is the key to achieve the clinical success of the restorations. Application of the surface coating material on the tooth structures will become a promising therapy in preventive dentistry in the future.

\section{Compliance with Ethics Guidelines}

Conflict of Interest Toru Nikaido, Go Inoue, Tomohiro Takagaki, Rena Takahashi, Alireza Sadr, and Junji Tagami declare that they have no conflict of interest.
Human and Animal Rights and Informed Consent This article does not contain any studies with human or animal subjects performed by any of the authors.

\section{References}

Papers of particular interest, published recently, have been highlighted as:

- Of importance

- Of major importance

1. Mount GJ, Ngo H. Minimal intervention: a new concept for operative dentistry. Quintessence Int. 2000;31(9):527-33.

2. Wassell RW, Walls AW, McCabe JF. Direct composite inlays versus conventional composite restorations: three-year clinical results. $\mathrm{Br}$ Dent J. 1995;179(9):343-9.

3. Umino A et al. Confocal laser scanning microscopic observations of secondary caries inhibition around different types of luting cements. Am J Dent. 2005;18(4):245-50.

4. Nikaido $\mathrm{T}$ et al. A resin coating technique to achieve minimal intervention in indirect resin composites: a case report. Int Chin J Dent. 2003;3(3):62-8.

5. Nikaido T et al. Protection and reinforcement of tooth structures by dental coating materials. Coatings. 2012;2:210-20.

6. Pashley EL, Caughman WF, et al. Dentin permeability: sealing the dentin in crown preparations. Oper Dent. 1992;17(1):13-20.

7. Kitasako $\mathrm{Y}$ et al. Histomorphometric analysis of dentinal bridge formation and pulpal inflammation. Quintessence Int. 2002;33(8): $600-8$.

8. Inokoshi S. Temporary sealing-pulp and dentin protection using low viscosity composite. Adhes Dent. 1992;10(2):250 (in Japanese).

9. Nikaido T et al. Effect of temporary filling materials on adhesion of dual cured resin cement to low viscosity resin. Jap J Dent Mater. 1993;12(6):655-61 (in Japanese).

10. Sato M et al. How to use "Liner Bond System" as a dentin and pulp protector in indirect restorations. Adhes Dent. 1994;12(2):41-8 (in Japanese).

11. Inokoshi $\mathrm{S}$ et al. Monkey pulpal response to adhesively luted indirect resin composite inlays. Oper Dent. 1995;20(3):111-8.

12. Momoi $\mathrm{Y}$ et al. Sealing ability of dentin coating using adhesive resin systems. Am J Dent. 2003;16(2):105-11.

13. Jayasooriya PR et al. The effect of a resin coating on the interfacial adaptation of composite inlays. Oper Dent. 2003;28(1):28-35.

14. Paul SJ, Schärer P. The dual bonding technique: a modified method to improve adhesive luting procedures. Int J Periodontics Restor Dent. 1997;17(6):536-45.

15. Bertschinger $\mathrm{C}$ et al. Dual application of dentin bonding agents: effect on bond strength. Am J Dent. 1996;9(9):115-9.

16. Magne P. Immediate dentin sealing: a fundamental procedure for indirect bonded restorations. J Esthet Restor Dent. 2005;17(3):14454.

17. Magne $\mathrm{P}$ et al. Immediate dentin sealing improves the bond strength of indirect restorations. J Prosthet Dent. 2005;94(6):511-9.

18. Van Meerbeek B et al. Technique-sensitivity of contemporary adhesives. Dent Mater J. 2005;24(1):1-13.

19. Nakabayashi N, Nakamura M, Yasuda N. Hybrid layer as a dentin bonding mechanism. J Aesthet Dent. 1991;3(4):133-8.

20. Tsuchiya $\mathrm{S}$ et al. Ultrastucture of the dentin-adhesive interface after acid base challenge. J Adhes Dent. 2004;6(3):183-90.

21. Inoue $\mathrm{G}$ et al. The acid-base resistant zone in three dentin bonding systems. Dent Mater J. 2009;28(6):717-21. 
22. Takagaki $\mathrm{T}$ et al. Effect of hybridization on bond strength and adhesive interface after acid-base challenge using 4-META/MMATBB resin. Dent Mater J. 2009;28(2):185-93.

23. Nurrohman $\mathrm{H}$ et al. Hydroxyapatite crystal protection against acidattack beneath resin-dentin interface with four adhesives: TEM and crystallography evidence. Dent Mater. 2012;28(7):e89-98. This manuscript represents the first description of the study on absence/presence of hydroxyapatite crystal at the bottom of the hybrid layer using different types of adhesive systems.

24. Inoue $\mathrm{S}$ et al. Hydrolytic stability of self-etch adhesives bonded to dentin. J Dent Res. 2005;84(12):1160-4.

25. Yoshida $\mathrm{Y}$ et al. Comparative study on adhesive performance of functional monomers. J Dent Res. 2004;83(6):454-8.

26. Yoshihara $\mathrm{K}$ et al. Nano-controlled molecular interaction at adhesive interfaces for hard tissue reconstruction. Acta Biomater. 2010;6(9):3573-82.

27. Van Meerbeek B et al. Correlative transmission electron microscopy examination of nondemineralized and demineralized resindentin interfaces formed by two dentin adhesive systems. J Dent Res. 1996;75(3):879-88.

28. Nikaido $\mathrm{T}$ et al. Assessment of the nanostructure of acid-base resistant zone by the application of all-in-one adhesive systems: Super dentin formation. Biomed Mater Eng. 2009;19(2-3):163-71.

29. Nikaido $\mathrm{T}$ et al. Tensile bond strengths of resin cements to bovine dentin using resin coating. Am J Dent. 2003;16(Spec):41A-6A.

30. Kitasako $\mathrm{Y}$ et al. Effect of resin-coating technique on dentin tensile bond strengths over 3 years. J Esthet Restor Dent. 2002;14(2):11522 .

31. Nikaido T et al. Effect of resin coating on dentin bond durability of a resin cement over 1 year. Am J Dent. 2008;21(1):64-8.

32. Jayasooriya PR et al. Efficacy of a resin coating on bond strengths of resin cement to dentin. J Esthet Restor Dent. 2003;15(2):105-13.

33. Belli $\mathrm{S}$ et al. The effect of additional enamel etching and a flowable composite to the interfacial integrity of class II adhesive composite restorations. Oper Dent. 2001;26(1):70-5.

34. Ariyoshi $\mathrm{M}$ et al. Microtensile bond strengths of composite cores to pulpal floor dentin with resin coating. Dent Mater J. 2008;27(3): 400-7.

35. Bista B et al. Nondestructive assessment of current one-step selfetch dental adhesives using optical coherence tomography. J Biomed Opt. 2013;18(7):076020. This manuscript represents the first description of the study of sealing performance of direct bonded restorations using a two-step and one-step self-etch adhesive systems by OCT.

36. Sun J, Eidelman N, Lin-Gibson S. 3D mapping of polymerization shrinkage using X-ray micro-computed tomography to predict microleakage. Dent Mater. 2009;25(3):314-20.

37. De Santis R et al. A 3D analysis of mechanically stressed dentinadhesive-composite interfaces using X-ray micro-CT. Biomaterials. 2005;26(3):257-70.

38. Sadr A et al. Swept source optical coherence tomography for quantitative and qualitative assessment of dental composite restorations. Proc SPIE. 2011;7884:78840C.

39. Nazari A et al. 3D assessment of void and gap formation in flowable resin composites using optical coherence tomography. J Adhes Dent. 2013;15(3):237-43.

40. Makishi $\mathrm{P}$ et al. Non-destructive 3D imaging of composite restorations using optical coherence tomography: marginal adaptation of self-etch adhesives. J Dent. 2011;39(4):316-25.

41.• Turkistani A et al. Sealing performance of resin cements before and after thermal cycling: evaluation by optical coherence tomography. Dent Mater. 2014;30(9):993-1004. This manuscript represents the first description of the study of sealing performance of indirect bonded restorations using the resin coating technique by OCT
42. Nikaido T et al. Evaluation of thermal cycling and mechanical loading on bond strength of a self-etching primer system to dentin. Dent Mater. 2002;18(3):269-75.

43. Takahashi $\mathrm{M}$ et al. Long-term evaluation of water sorption and ultimate tensile strength of HEMA-containing/-free one-step selfetch adhesives. J Dent. 2011;39(7):506-12.

44. Nakano $\mathrm{M}$ et al. Effect of impression material on adhesion of resin cement to resin coated teeth. J Jpn Adhes Dent. 1999;17(5):198 204(in Japanese)

45. Magne P, Nielsen B. Interaction between impression materials and immediate dentin sealing. J Prosthet Dent. 2009;102(5):298-305.

46. Hosaka $\mathrm{K}$ et al. Relationship between mechanical properties of onestep self-etch adhesives and water sorption. Dent Mater. 2010;26(4):360-7.

47. Magne P, Douglas WH. Porcelain veneers: dentin bonding optimization and biomimetic recovery of the crown. Int $\mathrm{J}$ Prosthodont. 1999; 12(2):111-21.

48. Nikaido $\mathrm{T}$ et al. The resin-coating technique. Effect of a single-step bonding system on dentin bond strengths. J Adhes Dent. 2003;5(4): 293-300.

49. Kosaka $\mathrm{S}$ et al. Effect of resin coating as a means of preventing marginal leakage beneath full cast crowns. Dent Mater J. 2005;24(1):117-22

50. Takahashi $\mathrm{R}$ et al. Thin resin coating by dual-application of all-inone adhesives improves dentin bond strength of resin cements for indirect restorations. Dent Mater J. 2010;29(5):615-22.

51. Maruoka $\mathrm{R}$ et al. Coronal leakage inhibition in endodontically treated teeth using resin-coating technique. Dent Mater J. 2006;25(1): $97-103$.

52. Horowitz HS, Heifetz SB, Poulsen S. Retention and effectiveness of a single application of an adhesive sealant in preventing occlusal caries: final report after five years of a study in Kalispell, Montana. J Am Dent Assoc. 1977;95(6):1133-2119.

53. Paris $\mathrm{S}$ et al. Progression of sealed initial bovine enamel lesions under demineralizing conditions in vitro. Caries Res. 2006;40(2): 124-9.

54. Phark JH et al. Caries infiltration with resins: a novel treatment option for interproximal caries. Compend Contin Educ Dent. 2009;30(3):13-7.

55. Schmidlin PR et al. Protection of sound enamel and artificial enamel lesions against demineralization: caries infiltrant versus adhesive. J Dent. 2012;40(10):851-6.

56. $\mathrm{Li} \mathrm{N}$ et al. The role of functional monomers in bonding to enamel: acid-base resistant zone and bonding performance. J Dent. 2010;38(9):722-30.

57.• Alsayed EZ et al. Optical coherence tomography for evaluation of enamel and protective coatings. Dent Mater J. 2015;34(1):98-107. This manuscript represents the study on enamel protection using an adhesive.

58. Heijnsbroek M, Paraskevas S, van der Weijden GA. Fluoride interventions for root caries: a review. Oral Health Prev Dent. 2007;5(2): $145-52$.

59. Kaneshiro AV et al. Effects of a self-etching resin coating system to prevent demineralization of root surfaces. Dent Mater. 2008;24(10): 1420-7.

60. Daneshmehr L et al. Effects of root dentin surface coating with allin-one adhesive materials on biofilm adherence. J Dent. 2008;36(1): 33-41.

61. Tajima $\mathrm{K}$ et al. Effects of coating root dentin surfaces with adhesive materials. Dent Mater J. 2009;28(5):578-86.

62.• Gando I et al. Resistance of dentin coating materials against abrasion by toothbrush. Dent Mater J. 2013;32(1):68-74. This manuscript represents the study on wear resistance of resin coating materials. 